International Journal of Pure and Applied Mathematics

Volume 104 No. 3 2015, 363-373

ISSN: $1311-8080$ (printed version); ISSN: 1314-3395 (on-line version)

url: http://www.ijpam.eu

doi: http://dx.doi.org/10.12732/ijpam.v104i3.7

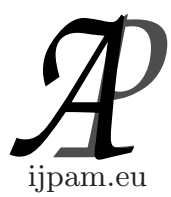

\title{
CERTAIN CLASSES OF MEROMORPHIC MULTIVALENT FUNCTIONS WITH RESPECT TO $(j, k)$-SYMMETRIC POINTS
}

\author{
C. Selvaraj ${ }^{1}$, K.R. Karthikeyan ${ }^{2}$, T.R.K. Kumar ${ }^{3} \S$ \\ ${ }^{1}$ Presidency College \\ Chennai, 600 005, Tamilnadu, INDIA \\ ${ }^{2}$ Salalah College of Technology \\ Salalah, SULTANATE OF OMAN \\ ${ }^{3}$ R.M.K. Engineering College \\ Kavaraipettai, 601206, Tamilnadu, INDIA
}

Abstract: The author introduces new class of $p$-valent meromorphic multivalent analytic function with respect $(j, k)$-symmetric points. Integral representation and inclusion relations for functions in these classes are obtained.

AMS Subject Classification: 30C45

Key Words: meromorphic, multivalent, $(j, k)$-symmetrical functions

\section{Introduction}

Let $\mathcal{M}_{p}$ denote the class of functions of the form

$$
f(z)=z^{-p}+\sum_{n=1}^{\infty} a_{n} z^{n-p}, \quad(p \geq 1),
$$

which are analytic in the punctured open unit disk

$$
\mathcal{U}^{*}=\{z: z \in \mathbb{C} \text { and } 0<|z|<1\}=\mathcal{U} \backslash\{0\} .
$$

Received: July 13, 2015

(C) 2015 Academic Publications, Ltd.

$\S_{\text {Correspondence author }}$ 
For the functions $f(z)$ of the form (1.1) and

$$
g(z)=z^{-p}+\sum_{n=1}^{\infty} b_{n} z^{n-p},
$$

the Hadamard product (or convloution) of $f$ and $g$ is defined by $(f * g)(z)=$ $z^{-p}+\sum_{n=1}^{\infty} a_{n} b_{n} z^{n-p}$.

For complex parameters $\alpha_{1}, \ldots, \alpha_{q}$ and $\beta_{1}, \ldots, \beta_{s}\left(\beta_{j} \in \mathbb{C} \backslash \mathbb{Z}_{0}^{-} ; \mathbb{Z}_{0}^{-}=\right.$ $0,-1,-2, \ldots ; j=1, \ldots, s)$, we define the generalized hypergeometric function ${ }_{q} F_{s}\left(\alpha_{1}, \ldots, \alpha_{q} ; \beta_{1}, \ldots, \beta_{s} ; z\right)$ by

$$
\begin{gathered}
{ }_{q} F_{s}\left(\alpha_{1}, \alpha_{2}, \ldots, \alpha_{q} ; \beta_{1}, \beta_{2}, \ldots, \beta_{s} ; z\right)=\sum_{n=0}^{\infty} \frac{\left(\alpha_{1}\right)_{n} \ldots\left(\alpha_{q}\right)_{n}}{\left(\beta_{1}\right)_{n} \ldots\left(\beta_{s}\right)_{n}} \frac{z^{n}}{n !} \\
\left(q \leq s+1 ; q, s \in \mathbb{N}_{0}=\mathbb{N} \cup\{0\} ; z \in \mathcal{U}\right),
\end{gathered}
$$

where $(x)_{k}$ is the Pochhammer symbol defined, in terms of the Gamma function $\Gamma$, by

$$
\begin{aligned}
(x)_{k} & =\frac{\Gamma(x+k)}{\Gamma(x)} \\
& = \begin{cases}1 & \text { if } k=0 \\
x(x+1)(x+2) \ldots(x+k-1) & \text { if } k \in \mathbb{N}=\{1,2, \ldots\} .\end{cases}
\end{aligned}
$$

Corresponding to a function $\mathcal{G}_{q, s}^{p}\left(\alpha_{1}, \beta_{1} ; z\right)$ defined by

$$
\mathcal{G}_{q, s}^{p}\left(\alpha_{1}, \beta_{1} ; z\right):=z^{-p} F_{s}\left(\alpha_{1}, \alpha_{2}, \ldots, \alpha_{q} ; \beta_{1}, \beta_{2}, \ldots, \beta_{s} ; z\right),
$$

we consider the operator $H^{p}\left(\alpha_{1}, \alpha_{2}, \ldots, \alpha_{q} ; \beta_{1}, \beta_{2}, \ldots, \beta_{s}\right) f: \mathcal{U} \longrightarrow \mathcal{U}$ which is defined by means of Hadamard product (or convolution):

$$
H^{p}\left(\alpha_{1}, \alpha_{2}, \ldots, \alpha_{q} ; \beta_{1}, \beta_{2}, \ldots, \beta_{s}\right) f(z)=\mathcal{G}_{q, s}^{p}\left(\alpha_{1}, \beta_{1} ; z\right) * f(z) .
$$

We observe that, for a function $f(z)$ of the form (1.1), we have

$$
\begin{aligned}
H^{p}\left(\alpha_{1}, \alpha_{2}, \ldots,\right. & \left.\alpha_{q} ; \beta_{1}, \beta_{2}, \ldots, \beta_{s}\right) f(z) \\
& =z^{-p}+\sum_{n=1}^{\infty} \frac{\left(\alpha_{1}\right)_{n} \ldots\left(\alpha_{q}\right)_{n}}{\left(\beta_{1}\right)_{n} \ldots\left(\beta_{s}\right)_{n}} \frac{a_{n} z^{n-p}}{n !} .
\end{aligned}
$$

For convenience, we write

$$
H^{p}\left(\alpha_{1}\right)=H^{p}\left(\alpha_{1}, \alpha_{2}, \ldots, \alpha_{q} ; \beta_{1}, \beta_{2}, \ldots, \beta_{s}\right) .
$$


It can be easily seen from (1.3) that

$$
z\left(H^{p}\left(\alpha_{1}\right) f(z)\right)^{\prime}=\alpha_{1} H^{p}\left(\alpha_{1}+1\right) f(z)-\left(\alpha_{1}+p\right) H^{p}\left(\alpha_{1}\right) f(z) .
$$

The linear operator $H^{p}\left(\alpha_{1}\right) f(z)$ was introduced and investigated by Liu and Srivastava [3].

Let $k$ be an arbitrarily fixed integer not smaller than 2 , then the set $\mathcal{G}_{k}$ of all roots of $k$-th degree of unity has the form

$$
\mathcal{G}_{k}=\left\{\varepsilon^{0}, \varepsilon^{1}, \ldots, \varepsilon^{k-1}\right\}
$$

where $\varepsilon=\exp (2 \pi i / k)$.

A function $f \in \mathcal{M}_{p}$ is said to be $(j, k)$-symmetrical if for each $z \in \mathcal{U}$

$$
\begin{gathered}
f(\varepsilon z)=\varepsilon^{-p j} f(z), \\
(k=1,2, \ldots ; j=0,1,2, \ldots(k-1)) .
\end{gathered}
$$

The family of $(j, k)$-symmetrical functions will be denoted by $\mathcal{F}_{k}^{j}$. We observe that $\mathcal{F}_{2}^{1}, \mathcal{F}_{2}^{0}$ and $\mathcal{F}_{k}^{1}$ are well-known families of odd functions, even functions and $k$-symmetrical functions respectively.

Also let $f_{j, k}(z)$ be defined by the following equality

$$
\begin{gathered}
f_{j, k}(z)=\frac{1}{k} \sum_{\nu=0}^{k-1} \frac{f\left(\varepsilon^{\nu} z\right)}{\varepsilon^{-\nu p j}}, \\
\left(f \in \mathcal{M}_{p} ; k=1,2, \ldots ; j=0,1,2, \ldots(k-1)\right) .
\end{gathered}
$$

It is obvious that $f_{j, k}(z)$ is linear from $\mathcal{U}$ into $\mathcal{U}$. The notion of $(j, k)$ symmetric functions was introduced and studied by P. Liczberski and J. Połubi'nski in [2].

If $\nu$ is an integer, then the following identities follow directly from (1.8):

$$
\begin{aligned}
& f_{j, k}\left(\varepsilon^{\nu} z\right)=\varepsilon^{-\nu p j} f_{j, k}(z), \\
& f_{j, k}^{\prime}\left(\varepsilon^{\nu} z\right)=\varepsilon^{-\nu p j-\nu} f_{j, k}^{\prime}(z)=\frac{1}{k} \sum_{\nu=0}^{k-1} \varepsilon^{\nu+\nu p j} f^{\prime}\left(\varepsilon^{\nu} z\right), \\
& f_{j, k}^{\prime \prime}\left(\varepsilon^{\nu} z\right)=\varepsilon^{-\nu p j-2 \nu} f_{j, k}^{\prime \prime}(z)=\frac{1}{k} \sum_{\nu=0}^{k-1} \varepsilon^{2 \nu+\nu p j} f^{\prime \prime}\left(\varepsilon^{\nu} z\right) .
\end{aligned}
$$


Throughout this paper, we assume that $p, k \in \mathbb{N}, \varepsilon=\exp (2 \pi i / k)$ and

$$
\begin{gathered}
f_{j, k}^{p}\left(\alpha_{1} ; z\right)=\frac{1}{k} \sum_{\nu=0}^{k-1} \varepsilon^{\nu j p}\left(H^{p}\left(\alpha_{1}\right) f\left(\varepsilon^{\nu} z\right)\right)=z^{-p}+\cdots, \\
\left(f \in \mathcal{M}_{p} ; k=1,2, \ldots ; j=0,1,2, \ldots(k-1)\right) .
\end{gathered}
$$

Clearly, for $j=k=1$, we have $f_{j, k}^{p}\left(\alpha_{1} ; z\right)=H^{p}\left(\alpha_{1}\right) f(z)$.

Motivated by the concept introduced by K. Sakaguchi in [6], recently several subclasses of analytic functions with respect to $k$-symmetric points were introduced and studied by various authors (see $[7,8,9,10])$. In this paper, new class of functions in $\mathcal{M}_{p}$ with respect to $(j, k)$-symmetric points is introduced.

For fixed parameters $A, B$ and $\lambda(-1 \leq B<A \leq 1 ; 0 \leq \lambda<p ; p \in$ $\mathbb{N})$, we say that a function $f(z) \in \mathcal{M}_{p}$ is in the class $\mathcal{S}_{j, k}^{p}\left(\alpha_{1} ; A, B ; \lambda\right)$ of meromorphically $p$-valent functions in $\mathcal{U}$, if it also satisfies the inequality:

$$
\left|\frac{\frac{z\left(H^{p}\left(\alpha_{1}\right) f(z)\right)^{\prime}}{f_{j, k}^{p}\left(\alpha_{1} ; z\right)}+p}{B \frac{z\left(H^{p}\left(\alpha_{1}\right) f(z)\right)^{\prime}}{f_{j, k}^{p}\left(\alpha_{1} ; z\right)}+[p B+(A-B)(p-\lambda)]}\right|<1, \quad(z \in \mathcal{U}) .
$$

If we let $j=k=1$ in $(1.11)$, the class $\mathcal{S}_{j, k}^{p}\left(\alpha_{1} ; A, B ; \lambda\right)$ reduces to the function class $\mathcal{Q}_{p, q, s}\left(\alpha_{1} ; A, B ; \lambda\right)$ introduced and studied by Aouf [1].

\section{Integral Representation}

Theorem 2.1. If $f \in \mathcal{S}_{j, k}^{p}\left(\alpha_{1} ; A, B ; \lambda\right)$, then

$$
f_{j, k}(z) \in \mathcal{Q}_{p, q, s}\left(\alpha_{1} ; A, B ; \lambda\right) .
$$

Proof. Let $f \in \mathcal{S}_{j, k}^{p}\left(\alpha_{1} ; A, B ; \lambda\right)$, then equivalently the condition (1.11) can be rewritten as have

$$
\frac{z\left(H^{p}\left(\alpha_{1}\right) f(z)\right)^{\prime}}{f_{j, k}^{p}\left(\alpha_{1} ; z\right)}=-\frac{p+[p B+(A-B)(p-\lambda)] w(z)}{1+B w(z)}
$$

where the function $w(z)$ is either analytic or meromorphic in $\mathcal{U}$, with $w(0)=0$. If we replace $z$ by $\varepsilon^{\nu} z$ in (2.1), then (2.1) will be of the form

$$
\frac{\varepsilon^{\nu} z\left(H^{p}\left(\alpha_{1}\right) f\left(\varepsilon^{\nu} z\right)\right)^{\prime}}{f_{j, k}^{p}\left(\alpha_{1} ; \varepsilon^{\nu} z\right)}=-\frac{p+[p B+(A-B)(p-\lambda)] w\left(\varepsilon^{\nu} z\right)}{1+B w\left(\varepsilon^{\nu} z\right)}
$$


Using (1.8) in (2.2), we get

$$
\frac{\varepsilon^{\nu+\nu p j} z\left(H^{p}\left(\alpha_{1}\right) f\left(\varepsilon^{\nu} z\right)\right)^{\prime}}{f_{j, k}^{p}\left(\alpha_{1} ; z\right)}=-\frac{p+[p B+(A-B)(p-\lambda)] w\left(\varepsilon^{\nu} z\right)}{1+B w\left(\varepsilon^{\nu} z\right)}
$$

Let $\nu=0,1,2, \ldots, k-1$ in $(2.3)$ respectively and summing them, we get

$$
\begin{aligned}
& \frac{\sum_{\nu=0}^{k-1} \varepsilon^{\nu+\nu p j} z\left(H^{p}\left(\alpha_{1}\right) f\left(\varepsilon^{\nu} z\right)\right)^{\prime}}{f_{j, k}^{p}\left(\alpha_{1} ; z\right)} \\
& \quad=-\frac{1}{k} \sum_{\nu=0}^{k-1} \frac{p+[p B+(A-B)(p-\lambda)] w\left(\varepsilon^{\nu} z\right)}{1+B w\left(\varepsilon^{\nu} z\right)} .
\end{aligned}
$$

Or equivalently,

$$
\frac{z\left(f_{j, k}^{p}\left(\alpha_{1} ; z\right)\right)^{\prime}}{f_{j, k}^{p}\left(\alpha_{1} ; z\right)}=-\frac{1}{k} \sum_{\nu=0}^{k-1} \frac{p+[p B+(A-B)(p-\lambda)] w\left(\varepsilon^{\nu} z\right)}{1+B w\left(\varepsilon^{\nu} z\right)}
$$

that is, $f_{j, k}(z) \in \mathcal{Q}_{p, q, s}\left(\alpha_{1} ; A, B ; \lambda\right)$.

Theorem 2.2. Let $f \in \mathcal{S}_{j, k}^{p}\left(\alpha_{1} ; A, B ; \lambda\right)$, then we have

$$
\begin{aligned}
& f_{j, k}^{p}\left(\alpha_{1} ; z\right) \\
& =z \exp \left\{\int_{0}^{\varepsilon^{\nu} z}\left(-\frac{1}{k} \sum_{\nu=0}^{k-1} \frac{p+[p B+(A-B)(p-\lambda)] w(t)}{t(1+B w(t))}-\frac{1}{t}\right) d t\right\},
\end{aligned}
$$

where $f_{j k}(z)$ defined by equality (1.8), $w(z)$ is analytic in $\mathcal{U}$ and $w(0)=0$, $|w(z)|<1$.

Proof. Let $f \in \mathcal{S}_{j, k}^{p}\left(\alpha_{1} ; A, B ; \lambda\right)$. In view of $(1.11)$, we have

$$
\frac{z\left(H^{p}\left(\alpha_{1}\right) f(z)\right)^{\prime}}{f_{j, k}^{p}\left(\alpha_{1} ; z\right)}=-\frac{p+[p B+(A-B)(p-\lambda)] w(z)}{1+B w(z)},
$$

where $w(z)$ is analytic in $\mathcal{U}$ and $w(0)=0,|w(z)|<1$. Substituting $z$ by $\varepsilon^{\nu} z$ in the equality $(2.5)$ respectively $\left(\nu=0,1,2, \ldots, k-1, \varepsilon^{k}=1\right)$, we have

$$
\frac{\varepsilon^{\nu} z\left(H^{p}\left(\alpha_{1}\right) f\left(\varepsilon^{\nu} z\right)\right)^{\prime}}{f_{j, k}^{p}\left(\alpha_{1} ; \varepsilon^{\nu} z\right)}=-\frac{p+[p B+(A-B)(p-\lambda)] w\left(\varepsilon^{\nu} z\right)}{1+B w\left(\varepsilon^{\nu} z\right)} .
$$


Using(1.8), equation (2.6) can be rewritten in the form

$$
\frac{z \varepsilon^{\nu+\nu p j}\left(H^{p}\left(\alpha_{1}\right) f\left(\varepsilon^{\nu} z\right)\right)^{\prime}}{f_{j, k}^{p}\left(\alpha_{1} ; z\right)}=-\frac{p+[p B+(A-B)(p-\lambda)] w\left(\varepsilon^{\nu} z\right)}{1+B w\left(\varepsilon^{\nu} z\right)} .
$$

Let $\nu=0,1,2, \ldots, k-1$ in $(2.7)$ respectively and summing them we get,

$$
\frac{z\left(f_{j, k}^{p}\left(\alpha_{1} ; z\right)\right)^{\prime}}{f_{j, k}^{p}\left(\alpha_{1} ; z\right)}=-\frac{1}{k} \sum_{\nu=0}^{k-1} \frac{p+[p B+(A-B)(p-\lambda)] w\left(\varepsilon^{\nu} z\right)}{1+B w\left(\varepsilon^{\nu} z\right)},
$$

From the equality (2.8), we get

$$
\frac{\left(f_{j, k}^{p}\left(\alpha_{1} ; z\right)\right)^{\prime}}{f_{j, k}^{p}\left(\alpha_{1} ; z\right)}-\frac{1}{z}=-\frac{1}{k} \sum_{\nu=0}^{k-1} \frac{p+[p B+(A-B)(p-\lambda)] w\left(\varepsilon^{\nu} z\right)}{z\left(1+B w\left(\varepsilon^{\nu} z\right)\right)}-\frac{1}{z} .
$$

Integrating this equality, we get

$$
\begin{aligned}
\log \{ & \left.\frac{f_{j, k}^{p}\left(\alpha_{1} ; z\right)}{z}\right\} \\
& =\int_{0}^{z}\left(-\frac{1}{k} \sum_{\nu=0}^{k-1} \frac{p+[p B+(A-B)(p-\lambda)] w\left(\varepsilon^{\nu} \zeta\right)}{\zeta\left(1+B w\left(\varepsilon^{\nu} \zeta\right)\right)}-\frac{1}{\zeta}\right) d \zeta \\
& =\int_{0}^{\varepsilon^{\nu} z}\left(-\frac{1}{k} \sum_{\nu=0}^{k-1} \frac{p+[p B+(A-B)(p-\lambda)] w(t)}{t(1+B w(t))}-\frac{1}{t}\right) d t
\end{aligned}
$$

or equivalently,

$$
\begin{aligned}
& f_{j, k}^{p}\left(\alpha_{1} ; z\right) \\
& =z \exp \left\{\int_{0}^{\varepsilon^{\nu} z}\left(-\frac{1}{k} \sum_{\nu=0}^{k-1} \frac{p+[p B+(A-B)(p-\lambda)] w(t)}{t(1+B w(t))}-\frac{1}{t}\right) d t\right\} .
\end{aligned}
$$

This completes the proof of Theorem 2.2.

Theorem 2.3. Let $f \in \mathcal{S}_{j, k}^{p}\left(\alpha_{1} ; A, B ; \lambda\right)$, then we have

$$
\begin{gathered}
H^{p}\left(\alpha_{1}\right) f(z) \\
=\int_{0}^{z} \exp \left\{\int_{0}^{\varepsilon^{\nu} \zeta}\left(-\frac{1}{k} \sum_{\nu=0}^{k-1} \frac{p+[p B+(A-B)(p-\lambda)] w(t)}{t(1+B w(t))}-\frac{1}{t}\right) d t\right\} \\
\cdot\left(-\frac{p+[p B+(A-B)(p-\lambda)] w(\zeta)}{1+B w(\zeta)}\right) d \zeta
\end{gathered}
$$

where $w(z)$ is analytic in $\mathcal{U}$ and $w(0)=0,|w(z)|<1$. 


\section{Inclusion Properties of the Class $\mathcal{S}_{j, k}^{p}\left(\alpha_{1} ; A, B ; \lambda\right)$}

We need the following Lemmas in the sequel.

Lemma 3.1. [4]Let $\beta(\beta \neq 0)$ and $\gamma$ be complex numbers and also let $\phi$ be convex and univalent in $\mathcal{U}$, with $\phi(0)=1$ and $\operatorname{Re}[\beta \phi(z)+\gamma]>0(z \in \mathcal{U})$. If $p(z)$ is analytic in $\mathcal{U}$ with $p(0)=\phi(0)$, then

$$
p(z)+\frac{z p^{\prime}(z)}{\beta p(z)+\gamma} \prec \phi(z) \quad \Longrightarrow \quad p(z) \prec \phi(z), \quad(z \in \mathcal{U}) .
$$

Lemma 3.2. [5]Let $\beta(\beta \neq 0)$ and $\gamma$ be complex numbers and also let $\phi$ be convex univalent in $\mathcal{U}$, with $\phi(0)=1$ and $\operatorname{Re}[\beta \phi(z)+\gamma]>0$. Also let $q(z) \prec \phi(z)$ If $p(z)$ is analytic in $\mathcal{U}$ with $p(0)=\phi(0)$, then

$$
p(z)+\frac{z p^{\prime}(z)}{\beta q(z)+\gamma} \prec \phi(z) \quad \Longrightarrow \quad p(z) \prec \phi(z), \quad(z \in \mathcal{U}) .
$$

Theorem 3.3. If

$$
\begin{gathered}
\Re\left\{\left(\alpha_{1}+p\right)-p\left(\frac{p+[p B+(A-B)(p-\lambda)] z}{1+B z}\right)\right\}>0, \\
(-1<B<A \leq 1 ; 0 \leq \lambda<p, p \in \mathbb{N}),
\end{gathered}
$$

then $\mathcal{S}_{j, k}^{p}\left(\alpha_{1}+1 ; A, B ; \lambda\right) \subset \mathcal{S}_{j, k}^{p}\left(\alpha_{1} ; A, B ; \lambda\right)$.

Proof. Let $f \in \mathcal{S}_{j, k}^{p}\left(\alpha_{1}+1 ; A, B ; \lambda\right)$ and set

$$
h(z)=-\frac{z\left(H^{p}\left(\alpha_{1}\right) f(z)\right)^{\prime}}{p f_{j, k}^{p}\left(\alpha_{1} ; z\right)}, \quad k(z)=-\frac{z\left(f_{j, k}^{p}\left(\alpha_{1} ; z\right)\right)^{\prime}}{p f_{j, k}^{p}\left(\alpha_{1} ; z\right)},
$$

we observe that $h(z)$ and $k(z)$ are analytic is $\mathcal{U}$ with $h(0)=k(0)=1$. Then by applying (1.5) in $h(z)$, we obtain

$$
h(z) f_{j, k}^{p}\left(\alpha_{1} ; z\right)=-\frac{\alpha_{1}}{p} H^{p}\left(\alpha_{1}+1\right) f(z)+\frac{\left(\alpha_{1}+p\right)}{p} H^{p}\left(\alpha_{1}\right) f(z) .
$$

Differentiating both sides of equation (3.3) with respect to $z$,

$$
z h^{\prime}(z)+\left(\left(\alpha_{1}+p\right)+\frac{z\left(f_{j, k}^{p}\left(\alpha_{1} ; z\right)\right)^{\prime}}{f_{j, k}^{p}\left(\alpha_{1} ; z\right)}\right)^{h(z)}
$$




$$
=-\frac{\alpha_{1}}{p} \frac{z\left(H^{p}\left(\alpha_{1}+1\right) f(z)\right)^{\prime}}{f_{j, k}^{p}\left(\alpha_{1} ; z\right)} .
$$

Using the relation between (1.5) and (1.10),

$$
\begin{aligned}
z\left(f_{j, k}^{p}\left(\alpha_{1} ; z\right)\right)^{\prime} & +\left(\alpha_{1}+p\right) f_{j, k}^{p}\left(\alpha_{1} ; z\right) \\
& =\frac{\alpha_{1}}{k} \sum_{\nu=0}^{k-1} \varepsilon^{-\nu j p}\left(H^{p}\left(\alpha_{1}+1\right) f\left(\varepsilon^{\nu} z\right)\right) \\
& =\alpha_{1} f_{j, k}^{p}\left(\alpha_{1}+1 ; z\right)
\end{aligned}
$$

Using (3.5) in (3.4), we have

$$
\begin{aligned}
h(z)+z h^{\prime}(z) & {\left[\left(\alpha_{1}+p\right)+\frac{z\left(f_{j, k}^{p}\left(\alpha_{1} ; z\right)\right)^{\prime}}{f_{j, k}^{p}\left(\alpha_{1} ; z\right)}\right]^{-1} } \\
& -\frac{z\left(H^{p}\left(\alpha_{1}+1\right) f(z)\right)^{\prime}}{p f_{j, k}^{p}\left(\alpha_{1}+1 ; z\right)} .
\end{aligned}
$$

From the definition of $\mathcal{S}_{j, k}^{p}\left(\alpha_{1}+1 ; A, B ; \lambda\right)$, we have

$$
h(z)+\frac{z h^{\prime}(z)}{\alpha_{1}+p-p k(z)} \prec \frac{p+[p B+(A-B)(p-\lambda)] z}{1+B z} .
$$

In view of the Lemma 3.2, the assertion of the Theorem would follow once we prove that $k(z) \prec \frac{p+[p B+(A-B)(p-\lambda)] z}{1+B z}(z \in \mathcal{U})$.

It follows from $k(z)$ and (3.5) that

$$
\left(\alpha_{1}+p\right)-p k(z)=-\alpha_{1} \frac{f_{j, k}^{p}\left(\alpha_{1}+1 ; z\right)}{p f_{j, k}^{p}\left(\alpha_{1} ; z\right)} .
$$

By logarithmical differentiation of equation (3.7), we get

$$
k(z)+\frac{z k^{\prime}(z)}{\left(\alpha_{1}+p\right)-p k(z)}=-\frac{z\left(f_{j, k}^{p}\left(\alpha_{1}+1 ; z\right)\right)^{\prime}}{p f_{j, k}^{p}\left(\alpha_{1}+1 ; z\right)} .
$$

Using Theorem 2.1 in the equality (3.8), we have

$$
k(z)+\frac{z k^{\prime}(z)}{\left(\alpha_{1}+p\right)-p k(z)} \prec \frac{p+[p B+(A-B)(p-\lambda)] z}{1+B z}
$$


In view of (3.1) and (3.9), we deduce from Lemma 2.1 that

$$
k(z) \prec \frac{p+[p B+(A-B)(p-\lambda)] z}{1+B z} .
$$

This implies that

$$
\mathcal{S}_{j, k}^{p}\left(\alpha_{1}+1 ; A, B ; \lambda\right) \subset \mathcal{S}_{j, k}^{p}\left(\alpha_{1} ; A, B ; \lambda\right) .
$$

Next we prove an inclusion property associated with a certain integral transform.

Theorem 3.4. Let $f \in \mathcal{M}_{p}$ and $F=L_{c}[f]$, where $L_{c}[f]$ is defined by

$$
L_{c}[f]=\frac{c-p}{z^{c}} \int_{0}^{z} t^{c-1} f(t) d t,\left(f \in \mathcal{M}_{p}\right) .
$$

If $f \in \mathcal{S}_{j, k}^{p}\left(\alpha_{1} ; A, B ; \lambda\right)$, then $F \in \mathcal{S}_{j, k}^{p}\left(\alpha_{1} ; A, B ; \lambda\right)$.

Proof. From definition of $F$ and the linearity of the operator $H^{p}\left(\alpha_{1}\right) f$, we have

$$
z\left(H^{p}\left(\alpha_{1}\right) F(z)\right)^{\prime}=(c-p) H^{p}\left(\alpha_{1}\right) f(z)-c H^{p}\left(\alpha_{1}\right) F(z) .
$$

From (3.11), we have

$$
(c-p) f_{j, k}^{p}\left(\alpha_{1} ; z\right)=c F_{j, k}^{p}\left(\alpha_{1} ; z\right)+z\left(F_{j, k}^{p}\left(\alpha_{1} ; z\right)\right)^{\prime}
$$

If we let

$$
w(z)=-\frac{z\left(F_{j, k}^{p}\left(\alpha_{1} ; z\right)\right)^{\prime}}{p F_{j, k}^{p}\left(\alpha_{1} ; z\right)},
$$

then $w$ is analytic in $\mathcal{U}$ and $p(0)=1$. From (3.12), we observe that

$$
c-p w(z)=(c-p) \frac{f_{j, k}^{p}\left(\alpha_{1} ; z\right)}{F_{j, k}^{p}\left(\alpha_{1} ; z\right)} .
$$

Differentiating both sides of (3.13) with respect to $z$, we obtain

$$
w(z)+\frac{z w^{\prime}(z)}{c-p w(z)}=-\frac{z\left(f_{j, k}^{p}\left(\alpha_{1} ; z\right)\right)^{\prime}}{p f_{j, k}^{p}\left(\alpha_{1} ; z\right)},
$$

By Theorem 2.1, we have

$$
w(z)+\frac{z w^{\prime}(z)}{c-p w(z)} \prec \frac{p+[p B+(A-B)(p-\lambda)] z}{1+B z}
$$


which on using Lemma 2.1 implies $w(z) \prec \frac{p+[p B+(A-B)(p-\lambda)] z}{1+B z}$. Now suppose that

$$
q(z)=-\frac{z\left(H^{p}\left(\alpha_{1}\right) F(z)\right)^{\prime}}{p F_{j, k}^{p}\left(\alpha_{1} ; z\right)}
$$

then $q(z)$ is analytic in $\mathcal{U}$, with $q(0)=1$, and it follows from (3.11) that

$$
F_{j, k}^{p}\left(\alpha_{1} ; z\right) q(z)=-\frac{(c-p)}{p} H^{p}\left(\alpha_{1}\right) f(z)+\frac{c}{p} H^{p}\left(\alpha_{1}\right) F(z) .
$$

Differentiating both sides of (3.14), we get

$$
z q^{\prime}(z)+(c-p w(z)) q(z)=-(c-p) \frac{z\left(H^{p}\left(\alpha_{1}\right) f(z)\right)^{\prime}}{p F_{j, k}^{p}\left(\alpha_{1} ; z\right)} .
$$

Now, from (3.13) and (3.15), we can deduce that

$$
\begin{gathered}
q(z)+\frac{z q^{\prime}(z)}{c-p w(z)}=-\frac{z\left(H^{p}\left(\alpha_{1}\right) f(z)\right)^{\prime}}{p F_{j, k}^{p}\left(\alpha_{1} ; z\right)} \\
\prec \frac{p+[p B+(A-B)(p-\lambda)] z}{1+B z} .
\end{gathered}
$$

Hence an application of Lemma 3.2 yeilds $q(z) \prec \frac{p+[p B+(A-B)(p-\lambda)] z}{1+B z}$, which shows that $F \in \mathcal{S}_{j, k}^{p}\left(\alpha_{1} ; A, B ; \lambda\right)$.

\section{References}

[1] M. K. Aouf, The generalized hypergeometric function and associated families of meromorphically multivalent functions, J. Math. Inequal. 3 (2009), no. $1,43-62$.

[2] P. Liczberski and J. Połubiński, On $(j, k)$-symmetrical functions, Math. Bohem. 120 (1995), no. 1, 13-28.

[3] J.L. Liu and H. M. Srivastava, Classes of meromorphically multivalent functions associated with the generalized hypergeometric function, Math. Comput. Modelling 39 (2004), no. 1, 21-34.

[4] S. S. Miller and P. T. Mocanu, On some classes of first-order differential subordinations, Michigan Math. J., 32 (1985), no. 2, 185-195. 
[5] Padmanabhan, K. S Parvatham, R. Some applications of differential subordination, Bull. Austral. Math. Soc. 32 (1985), no. 3, 321-330.

[6] K. Sakaguchi, On a certain univalent mapping, J. Math. Soc. Japan, 11 (1959), 72-75.

[7] Z.G. Wang, C.Y. Gao and S.-M. Yuan, On certain subclasses of close-toconvex and quasi-convex functions with respect to $k$-symmetric points, $J$. Math. Anal. Appl., 322 (2006), no. 1, 97-106.

[8] Z. G. Wang, Y. P. Jiang and H. M. Srivastava, Some subclasses of multivalent analytic functions involving the Dziok-Srivastava operator, Integral Transforms Spec. Funct. 19 (2008), no. 1-2, 129-146.

[9] D. G. Yang and J. L. Liu, On Sakaguchi functions, Int. J. Math. Math. Sci. 30 2003, 1923-1931.

[10] S. M. Yuan and Z. M. Liu, Some properties of $\alpha$-convex and $\alpha$-quasiconvex functions with respect to $n$-symmetric points, Appl. Math. Comput. 188 (2007), no. 2, 1142-1150. 
